$\mathbb{T}$ periodica polytechnica

Chemical Engineering

$53 / 2(2009) 61,66$

doi: 10.3311/pp.ch.2009-2.05

web: http://www.pp.bme.hu/ch

(c) Periodica Polytechnica 2009

RESEARCH ARTICLE

\section{Spectroscopy of laser-induced carbon plasma with nanosecond time resolution}

\author{
Balázs Nagy / László Nemes / Miklós Kubinyi
}

Received 2009-05-27

\begin{abstract}
Emission spectra in the visible spectral range generated by a $N d$ :YAG laser shining on graphite targets were taken with timeaveraging and time resolution. The analyses of the components and temperature of the plasma components were performed in helium environment using the LIBS (Laser Induced Breakdown Spectroscopy) technique.

The vibration-rotation temperature of the $C_{2}$ radical in the plasma initiates around $8000 \mathrm{~K}$ and drops exponentially with time. The rate coefficient of the exponential emission decay is $0.0018 \mathrm{~ns}^{-1}$. Thus, the expected lifetime of the $C_{2}$ Swan emission under our experimental conditions is $555 \mathrm{~ns}$.

In addition to $C_{2}$, singly and doubly ionized carbon, and in the first phase of the plasma, a continuum electron radiation was also observed.
\end{abstract}

\section{Keywords}

LIBS $\cdot$ carbon plasma $\cdot C_{2}$ radical $\cdot$ Swan bands $\cdot$ vibrationrotation temperature $\cdot \mathrm{Nd}: Y A G$ laser $\cdot$ graphite $\cdot$ plasma plume . nanosecond time resolution

\section{Acknowledgement}

The LIBS equipment was acquired from the Andor Technology Company, using funds from a European Project GVOP-3.2.12004-04-0059/3.0. We also acknowledge support from the Hungarian Scientific Research Fund OTKA (Grant NF72194).

\section{Balázs Nagy}

Institute of Structural Chemistry, Chemical Research Center, Hungarian Academy of Sciences, 1525 Budapest, P.O.B. 17, Hungary

\section{László Nemes}

Institute of Structural Chemistry, Chemical Research Center, Hungarian Academy of Sciences, H-1525 Budapest, P.O.B. 17, Hungary

\section{Miklós Kubinyi}

Department of Physical Chemistry and Materials Science, BME, H-1521, Budapest, Hungary

e-mail: kubinyi@chemres.hu

\section{Introduction}

The behaviour of carbon plasma has been studied from the second half of the 19th century, in the form of astronomical observations of comet spectra. Modern research on carbon plasmas may help in understanding fullerene and carbon nanotube production processes, their kinetics and molecular mechanisms, the way in which carbon clusters and subsequent forms of condensed phase carbon nanostructures, in particular thin films, are formed. As theory and experiment move forward in a synergistic and complementary way, electronic and vibrational properties probed with modern computer simulation methods promise insights into carbon dynamics. To some extent, the LIBS (Laser Induced Breakdown Spectroscopy) technique owes its widespread use to these carbon plasma applications. The description of the LIBS method is found in a number of sources, e.g. [1]-[3].

The LIBS method may be used to generate plasmas from any sample forms and phases. The emission spectra of these plasmas can be recorded with fast time resolution, and these spectra are then used to study the time evolution of plasma composition and temperature at nano- or picosecond resolution levels.

The LIBS method has been named this way due to the fact that laser photons ionize the media and in these ionized media volume electric discharges occur via dielectric breakdown processes. The components of the plasma are usually not in thermodynamic equilibrium and are usually highly excited. The energy required to break chemical bonds and ionize atoms is provided by the thermal and optical energy from the absorption and scattering of laser photons.

We have utilized the molecular emission Swan band of the $\mathrm{C}_{2}$ radical in the carbon plasma for vibrational-rotational plasma temperature measurements. Swan bands are a characteristic of the spectra of carbon stars, comets and of burning hydrocarbon fuels. They are named after the Scottish physicist William Swan (1818-1894) who first studied the spectral analysis of $C_{2}$ in 1856 [4]. This band corresponds to a triplet-triplet electronic transition. The spectroscopy of the $\mathrm{C}_{2}$ radical is very extensive, e.g. [5]-[8]. 


\section{Experiments}

The experimental setup is displayed in Fig. 1. We used a stationary graphite target for plasma generation and a $10 \mathrm{~Hz}$ flash lamp pumped Nd:YAG laser for generating the plasma. The laser beam was narrowed using a two lens inverted telescope to increase laser fluence on the sample surface. In using the $1064 \mathrm{~nm}$ laser radiation with telescopic beam narrowing and electronic pulse energy reduction the typical fluence was about 150 megawatt $/ \mathrm{cm}^{2}$, while using the $532 \mathrm{~nm}$ laser radiation this figure was around 60 megawatt $/ \mathrm{cm}^{2}$.

The graphite sample was placed inside a cylindrical quartz plasma cuvette that could be evacuated and filled with a background gas. In these experiments, we used helium as background gas at 50 Torr pressure. The emitted plasma light was collected by a multicomponent optical fiber perpendicularly to the cuvette axis. On the receiving end of the fiber a small quartz lens collected light from a wide angle: our spectra were thus spatially averaged. At the end of the fiber bundle the individual fibers were arranged in a plane to fit the entrance slit of the spectrograph. Two gratings were used, a lower resolution one with 150 lines/mm and blazing angle at $500 \mathrm{~nm}$, and a 1200 lines/mm grating blazed at $300 \mathrm{~nm}$. Scattered laser light from the $532 \mathrm{~nm}$ laser radiation was blocked by a notch filter. Occasionally light below $300 \mathrm{~nm}$ was also filtered out by a high pass filter, removing light below $300 \mathrm{~nm}$. In this way 2 nd order spectral lines arising from the UV region were removed.

Emission spectra were observed using a triple grating spectrograph of Czerny-Turner arrangement and a focal length of $303 \mathrm{~mm}$, equipped with a water-cooled $\left(-35^{\circ} \mathrm{C}\right)$ intensified Andor IStar DH 720-18U-03 ICCD detector. An electronic gain factor of 50 was generally employed (the maximum gain factor is 255), and an entrance slitwidth of 50 micron. Ten spectra were accumulated to increase signal to noise ratio. More extensive accumulation would have resulted in fast blackening of the cuvette walls. Occasionally these parameters were changed to optimize the intensity and signal to noise of the spectra, and also to minimize blackening of the LIBS cuvette walls by the ablated material from the graphite target.

Emission spectra were wavelength calibrated either by using the built-in automatic wavelength calibration function or by using a Pen-Ray low pressure cold cathode Mercury-Argon lamp and processed by the Andor proprietary Solis software. The automatic calibration is faster but less accurate than using the calibration lamp.

\subsection{Spectroscopic measurements}

First spectra were taken using the $1064 \mathrm{~nm}$ fundamental Nd:YAG laser wavelength. The ICCD detector was optically aligned with the fiber entrance slit to produce the sharpest and most symmetric atomic lines from the $\mathrm{Hg}$-Ar calibration lamp. Laser pulse energy was set to an optimal value that provided strong enough emission but did not lead to extensive ablation of the graphite target. Then exploratory low resolution time-

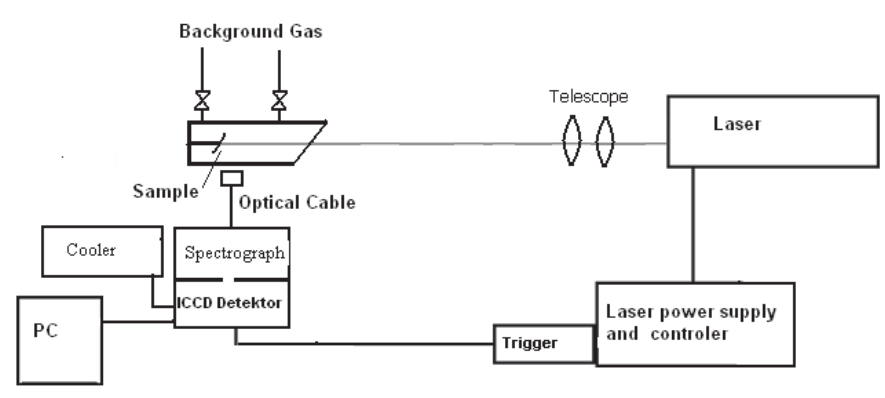

Fig. 1. Experimental LIBS arrangement

resolved spectra were taken using the 150 grooves/mm grating that provided the widest wavelength range and shortest running time, in order to minimize the deposition of amorphous carbon on the cuvette walls. Thereafter higher resolution spectra were taken using the 1200 grooves/mm grating at appropriate time delay values. These higher resolution spectral details had a range of $40 \mathrm{~nm}$, and these ranges were joined into a wider spectrum using the so-called 'Step and Glue' technique provided by the proprietary Andor Solis software.

The expected lifetimes for excited atomic and molecular states were determined using time-resolved LIBS methods. The decay of excited levels usually follows first-order, single exponential kinetics. It is therefore easy to determine the rate coefficient for the decay by using a logarithmic plot of emission intensities $I$ against time

$$
\ln I=\ln I_{0}-k t
$$

The reciprocal of the decay coefficient $k$ gives the expected lifetime of the emitting species.

To generate data for the plot in Eq. (1) we used the 'Step and Glue' method to collect emission spectra time-slices at 80 ns intervals, using $532 \mathrm{~nm}$ laser radiation for plasma excitation. Each time-slice spectrum was fit to exact theoretical $\mathrm{C}_{2}$ Swan band vibration-rotation intensities by means of the NMT (Nelder-Mead Temperature) algorithm to obtain a local thermal equilibrium vibration-rotation temperature estimate [9].

The NMT code is based on computing molecular emission intensities and thermal equilibrium level populations by the equations:

$$
I_{u l}=h v_{u l} A_{u l} N_{u}
$$

and

$$
N_{u}=N_{0} \frac{g_{u} e^{\frac{-E_{u}}{k T}}}{Q}
$$

where $h$ is Planck's constant, $v_{u l}$ is the spectral line frequency for radiative decay of the upper state $u$ to the lower state $l ; A_{u l}$ is the Einstein transition probability (emission coefficient) for the $u \rightarrow l$ transition; $N_{u}$ is the population density of upper state $u$; $N_{0}$ is the total population density of radiating species; $g_{u}$ is the statistical weight (degeneracy) of the upper state $u ; k$ is Boltzmann's constant; $T$ is the absolute temperature; $E_{u}$ is the energy of the upper state $u$ and $Q$ is the partition function. 
The condition for overall thermal equilibrium is frequently not realized in plasmas that tend to be off-equilibrium, but at high enough electron densities the equilibrium condition can still be realized for small spatial domains.

The determination of vibration-rotation temperature starts with an initial temperature guess. The calculated spectrum is compared to the experimental one and the sum of squared residuals is reduced by an appropriate temperature change. The fitting process continues in steps until the error of the fit becomes smaller than a given limit. The least-squares process is carried out corresponding to the Nelder-Mead Temperature (NMT) algorithm [9], while the calculation of the theoretical spectrum is based on computed diatomic line-strength (Hönl-London) factors [10].

\section{Results}

\subsection{Time-averaged carbon plasma spectra}

Fig. 2] shows a typical time-averaged spectrum taken with the 1200 lines/mm grating. Some singly ionized carbon atomic lines and some partially resolved Swan molecular bands of the $C_{2}$ radical are indicated in the figure.

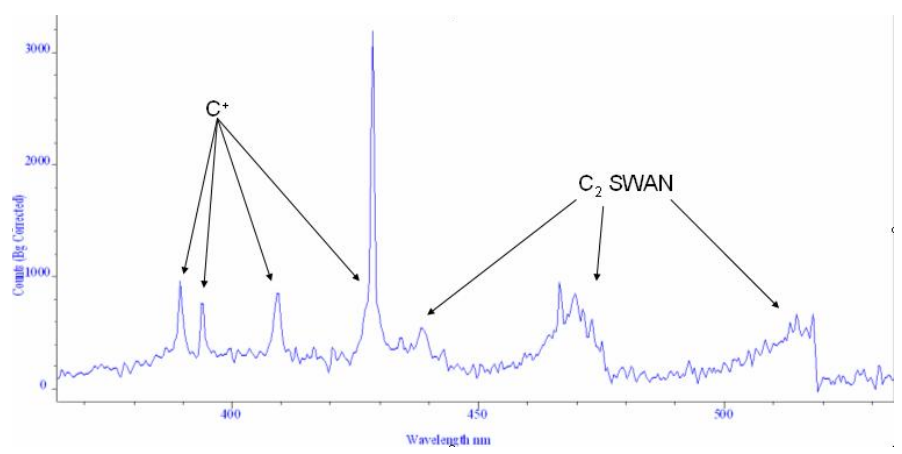

Fig. 2. Time-averaged carbon plasma emission spectrum taken in vacuo

Time-averaged spectra, like the one above, are more intense and therefore have a better signal-to-noise ratio than time resolved spectra, to be discussed later. Relatively weak atomic lines are easier to identify in time averaged spectra. For this reason we have always used time averaged operation before taking kinetic spectra. One serious disadvantage of time averaging is that spectroscopic temperature determination leads to badly determined estimates as lines and molecular features are integrated with continuously varying conditions during the cooling of the plasma.

\subsection{Low spectral resolution time-resolved (kinetic) spectra}

Kinetic spectra were taken using the time-resolution capabilities of the Andor ICCD detector. Time resolution is achieved by electronic gating of the image intensifier. The highest available time resolution is $2 \mathrm{~ns}$. However, the Nd:YAG laser pulse limits time resolution to a minimum of 6-7 ns due to the laser pulse width. In addition, we used an electronic method to reduce the laser pulse energy by modifying the trigger offset between the flash lamp and the active Q-switch. As a result our time resolution is limited to about 10-12 ns.

Running kinetic spectra involves taking individual spectra at selectable time intervals using preset time-gating parameters. Since we were interested in spectra in wide wavelength ranges and since kinetic spectra cannot be taken using the 'Step and Glue' function, we were limited to using the lowest resolution grating in order to cover a wider spectral range. Lower resolution spectra can be taken faster than those by the 'Step and Glue' technique and thus the experiments were easier to carry out thanks to reduced blackening of the cuvette walls by carbon ablates. However higher spectral resolution is needed for the characterization of atomic and molecular features. This problem was solved by applying a two-step procedure. First, kinetic runs using the 150 lines/mm grating were taken to determine the gating parameters suitable to record meaningful spectra, and in the second step single spectra were run using the 1200 lines/mm grating and 'Step and Glue' technique at preset timing points.

In choosing the timing parameters there are two considerations. The starting point of the plasma evolution is the arrival of the laser pulse, and the end time-point is chosen when the spectral features become weak due to the decay of the plasma. When the $532 \mathrm{~nm}$ laser radiation was used and scattered laser light was blocked by the $532 \mathrm{~nm}$ notch filter, the starting point of the plasma evolution was established by the appearance of the broad Bremsstrahlung emission due to electron radiation from the plasma [11]. As this emission occurs at the very beginning of the plasma evolution, it serves as a convenient starting point for the kinetic runs. The same method was applied when $1064 \mathrm{~nm}$ light was used, as the scattered laser light is not detected by the ICCD since the photocathode is not sensitive at this wavelength. The starting time of the kinetic spectra was again indicated by the appearance of the broad Bremsstrahlung radiation.

In Fig. 3.a. a kinetic series of spectra is shown. The wavelength of the laser was $1064 \mathrm{~nm}$, the gate width was $50 \mathrm{~ns}, 100$ ns time steps were used, and the laser beam was fully collimated to $2 \mathrm{~mm}$ diameter. At the start of the run the cuvette walls were already blackened by carbon deposition from a previous experiment.

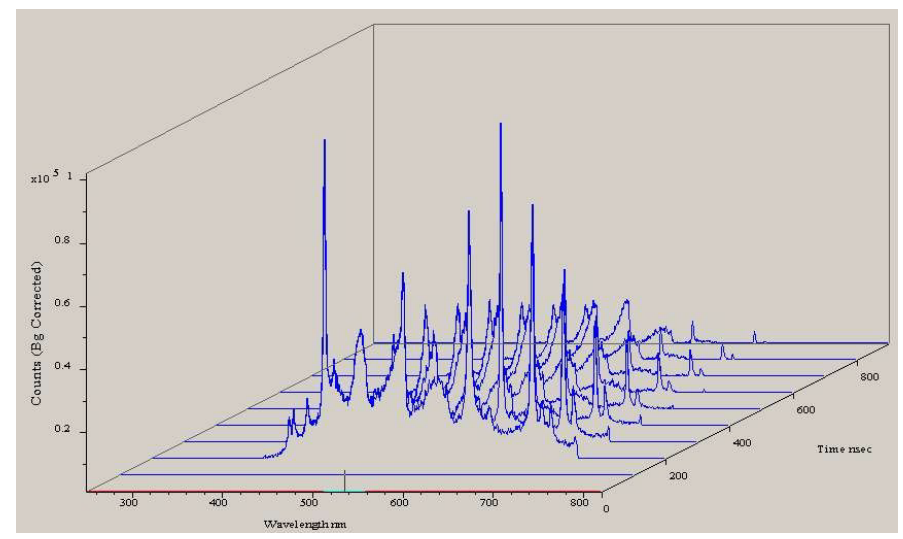

Fig. 3.a. Carbon plasma kinetics taken by the $1064 \mathrm{~nm}$ laser 


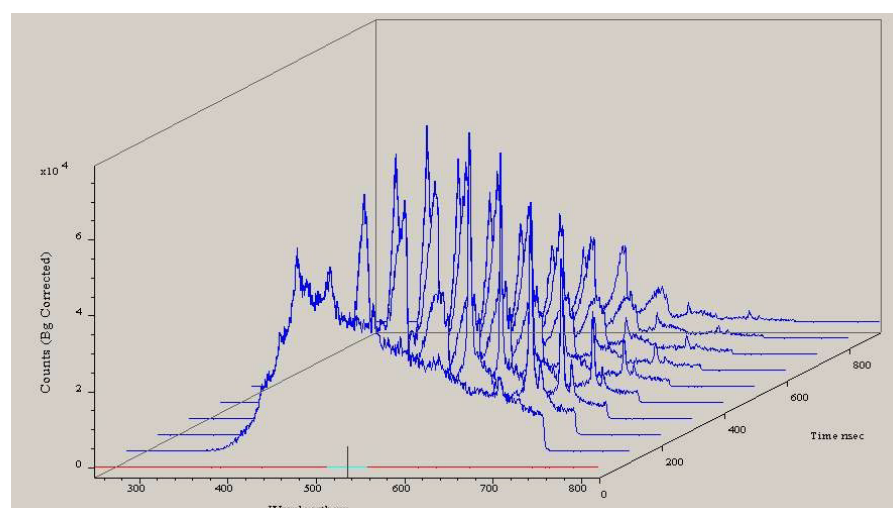

Fig. 3.b. Carbon kinetic spectra initiated at the emergence of the Bremsstrahlung radiation

In Fig. 3.b the collection of kinetic runs was started somewhat earlier than in Fig. 3.a In this way the broad Bremsstrahlung radiation could also be seen. The $426.7 \mathrm{~nm}$ ionized carbon line is much stronger in Fig. 3.a than in Fig. 3.b. This may be due to a difference between the blackening of the cuvette walls in the two experiments, as the amorphous carbon deposit strongly absorbs deep blue and uv radiation.

The various atomic spectral features were identified with the help of the NIST atomic spectral database [12], while broad features between 460 and $580 \mathrm{~nm}$ are due to the Swan bands of the $\mathrm{C}_{2}$ radical. Table 1 contains the spectral assignments.

\subsection{Decay rate coefficient fits}

Using the relative intensities of the spectral features in the various spectral slices from the low resolution kinetic runs, natural logarithmic plots were made against time delays. Relative intensities were normalized to the highest intensity features in the spectral slices. The slope of these plots yields the decay rate constant. This is the correct procedure when the time decay follows first-order kinetics. The decay plots are given in Fig. 4 for different spectral features. The quality of these plots (see the correlation coefficients) proves that the decay indeed corresponds to a single exponential process.

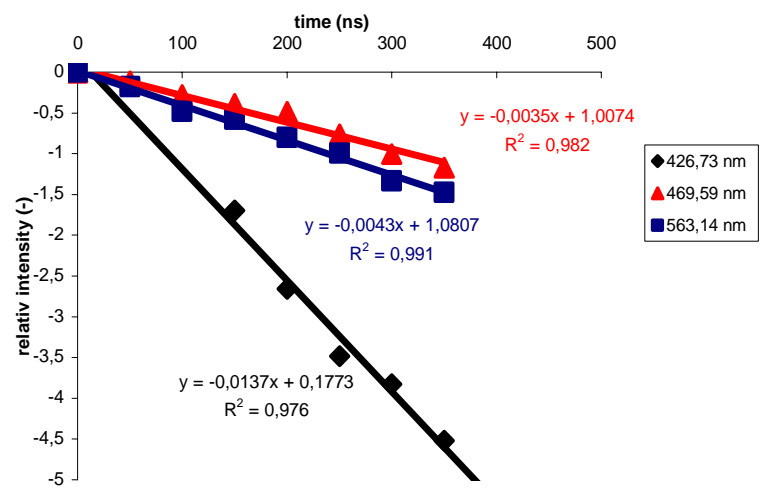

Fig. 4. Time decay of spectral emission at various wavelengths

As can be seen in Table 1 there are three essentially different values of the decay rate coefficients. The singly ionized carbon line decays with a $k$ value of $0.0129-0.0137 \mathrm{~ns}^{-1}$, the neutral he- lium line decays with $0.0045 \mathrm{~ns}^{-1}$, while the various $\mathrm{C}_{2}$ molecular features decay with $k$ values between 0.0031 and 0.0046 $\mathrm{ns}^{-1}$. The reciprocal values of these rate coefficients are then: C II: 73-78 ns, He I: 222 ns, while for the $C_{2}$ Swan features: 217-323 ns. These reciprocal values correspond to mean decay lifetimes.

Tab. 1. Assignments and decay rate constants of spectral features

\begin{tabular}{llll}
\hline $\begin{array}{l}\text { Spectral } \\
\text { wavelength } \\
(\mathrm{nm})\end{array}$ & $\begin{array}{l}\text { Spectral } \\
\text { assignment }\end{array}$ & $\begin{array}{l}\text { Rate } \\
\text { coefficient } \\
{[1 / \mathrm{ns}]}\end{array}$ & $\begin{array}{l}\text { Correlation } \\
\text { coefficient } \\
\mathrm{R}^{2}\end{array}$ \\
\hline 426.73 & $\mathrm{C} \mathrm{II}_{2}$ & 0.0137 & 0.976 \\
469.59 & $\mathrm{C}_{2}$ & 0.0035 & 0.982 \\
471.32 & $\mathrm{C}_{2}$ & 0.0035 & 0.993 \\
473.42 & $\mathrm{C}_{2}$ & 0.0034 & 0.995 \\
516.21 & $\mathrm{C}_{2}$ & 0.0031 & 0.904 \\
553.72 & $\mathrm{C}_{2}$ & 0.0046 & 0.985 \\
558.12 & $\mathrm{C}_{2}$ & 0.0045 & 0.963 \\
563.14 & $\mathrm{C}_{2}$ & 0.0043 & 0.991 \\
587.56 & $\mathrm{He} \mathrm{I}_{609.53}^{\mathrm{C} \mathrm{II}}$ & 0.0045 & 0.995 \\
\hline
\end{tabular}

\subsection{Time dependence of the $\mathrm{C}_{2}$ vibration-rotation tempera- ture}

The spectra that were recorded with the 1200 lines/mm grating were spectral slices between 410 and $640 \mathrm{~nm}$, obtained by applying the 'Step and Glue' technique using $40 \mathrm{~nm}$ wide spectral portions. These spectral slices were taken at predetermined delay times and were used for NMT temperature fits. We estimate the accuracy of NMT fits to $\mathrm{C}_{2}$ Swan vibration-rotation temperature to be around 200-300 K.

Recording the carbon plasma emission from plasmas excited by the $532 \mathrm{~nm}$ laser radiation yielded greater $\mathrm{C}_{2}$ Swan band intensities with good signal to noise ratio. This is due to the fact that using the green laser light led to less thermal effects and reduced ablation relative to the $1064 \mathrm{~nm}$ laser radiation, thus allowed longer spectral run times.

Figs. $5 \mathrm{a}$-b show the differences between time slices taken at time delays 100-180 ns and 260-340 ns, relative to the Bremsstrahlung radiation, the temperature fits are shown in red. The experimental spectra (in black) exhibit a hole around the $532 \mathrm{~nm}$ laser wavelength due to the use of the notch filter. These areas were excluded from the temperature fit.

The results of the NMT fits to the $\mathrm{C}_{2}$ Swan band vibrationrotation temperature are summarized in Table 2 Fig. 6 shows the natural logarithmic plot of these temperatures against time. The temperature change can be characterized by a single exponential with a decay coefficient of $0.0018 \mathrm{~ns}^{-1}$. The corresponding mean lifetime is $556 \mathrm{~ns}$.

\section{Disscusion}

It is an interesting observation that the time decay of the vibration-rotation temperature of the $\mathrm{C}_{2}$ Swan bands is similar 


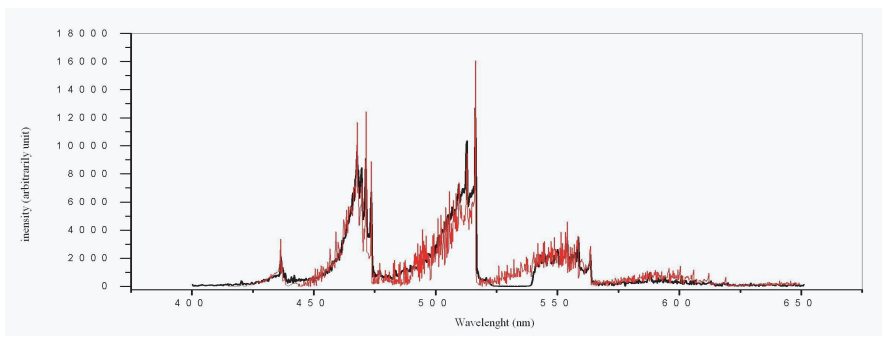

a.

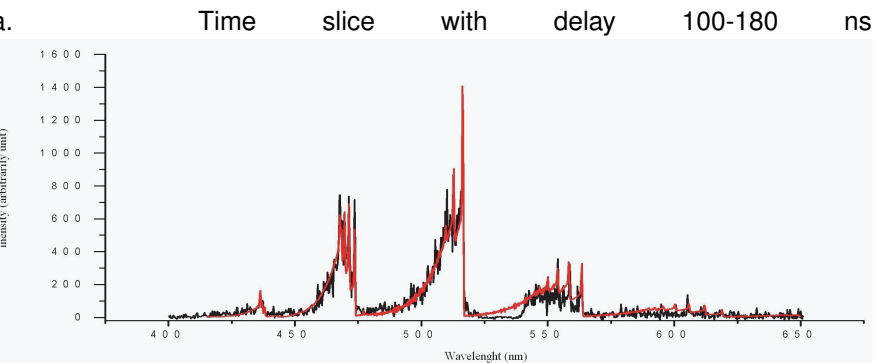

b. Time slice with delay $260-340 \mathrm{~ns}$

Fig. 5. Experimental (black) and fitted spectra (red)

Tab. 2. Vibration-rotation temperature values from NMT fits

\begin{tabular}{lll}
\hline $\begin{array}{l}\text { Time interval } \\
{[\mathrm{ns}]}\end{array}$ & $\begin{array}{l}\text { Interval centre } \\
{[\mathrm{ns}]}\end{array}$ & $\begin{array}{l}\text { Vib-rot } \\
\text { temperature } \\
{[\mathrm{K}]}\end{array}$ \\
\hline $20-100$ & 60 & 8777.2 \\
$100-180$ & 140 & 7319.8 \\
$180-260$ & 220 & 6515.0 \\
$260-340$ & 300 & 5594.8 \\
$340-420$ & 380 & 4948.0 \\
\hline
\end{tabular}

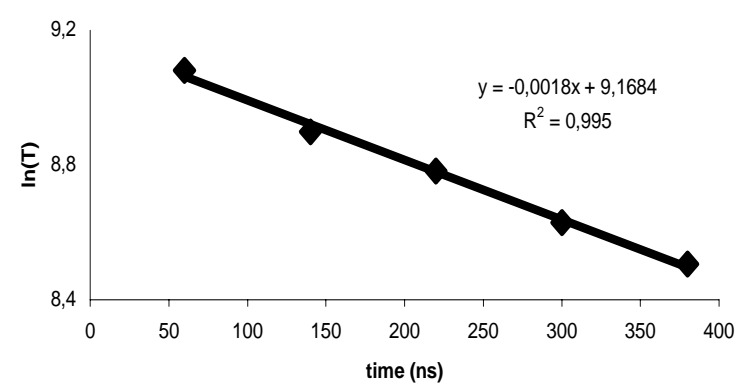

Fig. 6. The exponential fit to the time decay of $\mathrm{C}_{2}$ Swan vib-rot temperature to the time decay of the emission intensities of various features within the Swan bands. The intensity of emission depends on both the population of the upper (emitting) level and the temperature (see Eqs. 2 and 3). Thus, the time dependence of the temperature and the time dependence of emission intensity are correlated but not identical. These decays are not identical as the total emission intensity depends obviously also on the number of the emitting molecules. In order to determine the population of the emitting levels one would have to measure the absolute emission intensities. This complicated and difficult analysis was not undertaken in the present work.

However, as the collisionless lifetime of the $C_{2}$ Swan levels is around $115 \mathrm{~ns}[14]$ our observation of a significantly longer mean emission lifetime indicates that $\mathrm{C}_{2}$ is continously produced in the excited upper states of the Swan bands in the laserinduced plasma. The most probable mechanism for $\mathrm{C}_{2}$ production in this emitting state is radiative recombination of electronically excited neutral carbon atoms [15, 16].

We should add that our NMT temperature fits to the $\mathrm{C}_{2}$ Swan bands in Fig. 5 are subject to model errors. There are at least three model errors involved. First, laser-induced plasmas are never in spatially extensive thermal equilibrium. Only local thermal equilibrium (LTE) is possible. Secondly, our emission spectra were not corrected for the spectral variation of the optical transfer function of the combined spectrograph/ICCD system. The most important source of this spectral variation is the spectral sensitivity of the photocathode of the ICCD. We plan to carry out such relative intensity calibrations in the future. Finally, our spectra are spatially averaged and thus correspond to a collection of $\mathrm{C}_{2}$ molecules in various spatial regions with different particle number densities and plasma chemical composition.

Table 1 shows that atomic features in our spectra have mean emission lifetimes different from those of $\mathrm{C}_{2}$ Swan features. This difference also provides a possibility for distinguishing atomic and molecular features in plasma spectra. This is especially important in congested spectral regions rich in molecular features, such as the rotational structure of diatomic molecular bands.

\section{References}

1 Miziolek A W, Palleschi V, Schechter I (eds.), Laser Induced Breakdown Spectroscopy, Cambridge University Press, New York, 2006.

2 Fang-Yu Yueh, Jagdish P. Singh, Hansheng Zan, Encyclopedia of Analytical Chemistry, John Wiley and Sons Ltd., 2000.

3 Hahn D W, Miziolek A W, Palleschi V, Appl. Opt. 42 (2003), 5937.

4 Swan W, Trans. Roy.Soc. Edinburgh 21 (1857), 411-430.

5 Johnson R C, Phil.Trans. Roy.Soc. London, Series 226 (1927), 157-230.

Pretty W E, Proc. Phys. Soc. 40 (1927), 71-78.

7 Hopkins J, Glossary of Astronomy and Astrophysics, Univ.Chigao Press, 2001.

8 Gaydon A G, The Spectroscopy of Flames, Chapman and Hall, London, 1974. 2nd Ed.

9 Hornkohl J O, Parigger C G, Lewis J W L, J. Quant. Spectrosc. Radiat. Transfer 46 (1991), 405.

10 Hornkohl J O, Parigger C G, Nemes L, Appl.Opt. 44 (2005), 3686-3695. 
11 Harilal S S, Bindhu C V, Issac R C, Nampoori V P N, Vallabhan C P G, J. Appl. Phys. 82 (1997), 2140-2146.

12 available at http://physics.nist.gov/PhysRefData/ASD/lines_ form.html

13 Gaydon A G, The Spectroscopy of Flames, Chapman and Hall, London, 1974.

14 Wakasaki T, Jpn. J.Appl.Phys. Part 2, Letters 41 (2002), 5792-5796.

15 Sakka T, Iwanaga S, Ogata Y H, Matsunawa A, Takemoto T, J.Chem. Phys. 112 (2000), 8645-8653.

16 Osipov V V, Solomonov V I, Platonov V V, Snigereva O A, Ivanov M G, Lisenkov V V, Quantum. Electr. 35 (2005), 467-473. 